\title{
Hand grip strength predicts cardiovascular risk
}

G rip strength-the force exerted when an individual squeezes an object as tightly as possible with their hands-is an easy, quick, and cheap way to measure muscle strength. In an analysis of data for almost 140,000 individuals enrolled in the multinational Prospective Urban-Rural Epidemiology (PURE) study, grip strength was found to be as good a predictor of cardiovascularrelated mortality as systolic blood pressure, and a better predictor of all-cause mortality. The PURE study investigators point out that their findings are "widely generalisable", because their study "was done in a large cohort of participants from various countries, ethnic origins, and socioeconomic backgrounds".

Reduced grip strength, and therefore muscle strength, has previously been linked to increased risk of death, but the data for this association have mainly been derived from studies of individuals residing in high-income countries. The PURE study is a large, prospective cohort study being conducted in 17 high-income, middle-income, and lowincome countries around the world. Grip strength was measured using a Jamar dynamometer in the 139,691 study participants included in this particular analysis. Outcomes were assessed after a median follow-up duration of 4 years.

In total, 3,379 (2.4\%) study participants died during follow-up. Grip strength was inversely associated with all-cause death (HR 1.16, 95\% CI 1.13-1.20, $P<0.0001$ ), death from a cardiovascular cause (HR 1.17, 95\% CI 1.11-1.24, $P<0.0001$ ), death not attributable to a cardiovascular cause (HR 1.17, 95\% CI 1.12-1.21, $P<0.0001)$, myocardial infarction (HR 1.07, 95\% CI 1.02-1.11, $P=0.0024$ ), and stroke (HR 1.09, 95\% CI 1.05-1.15, $P<0.0001)$. Grip strength was not associated with various other conditions, such as diabetes mellitus or respiratory disease. The inverse relationship between grip strength and all-cause death, and between grip strength and death from

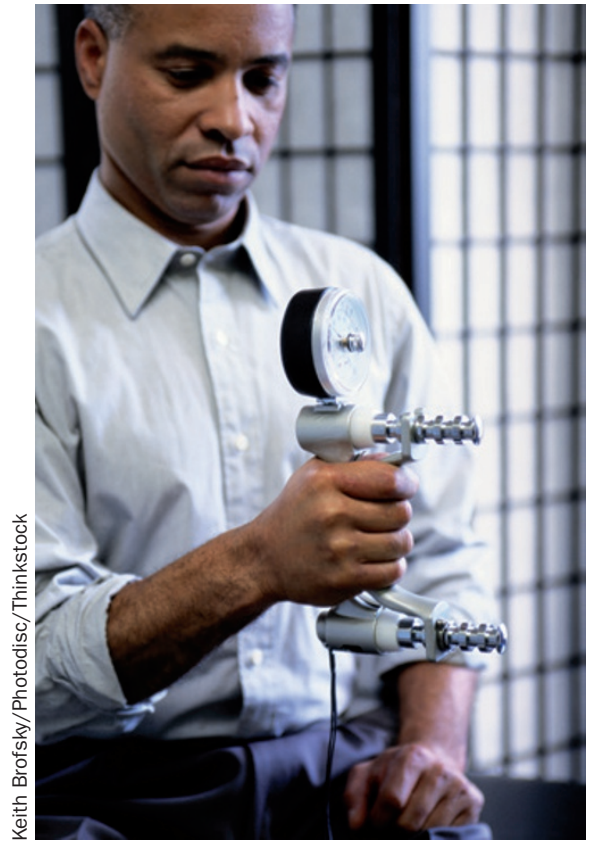

a cardiovascular cause, was consistent across sex and age.

The PURE investigators performed a post-hoc comparison of the prognostic value of grip strength, systolic blood pressure, and physical activity levels. Grip strength (HR per SD reduction 1.37, 95\% CI 1.28-1.47, $P<0.0001$ ) was found to be a stronger predictor of all-cause death than both systolic blood pressure (HR per SD increase 1.15, 95\% CI $1.10-1.21, P<0.0001)$ and physical activity (HR per SD reduction 1.09, 95\% CI 1.04-1.15, $P=0.002)$. For cardiovascularrelated death, the predictive value of grip strength (HR per SD reduction $1.45,95 \%$ CI $1.30-1.63, P<0.0001)$ was found to be similar to that of systolic blood pressure (HR per SD increase $1.43,95 \%$ CI $1.32-1.57, P<0.0001)$ and stronger than that of physical activity (HR per SD reduction 1.12, 95\% CI 1.03-1.22, $P=0.01)$. For cardiovascular disease, the predictive value of grip strength (HR per SD reduction $1.21,95 \%$ CI $1.13-1.29$, $P<0.0001)$ was found to be weaker than that of systolic blood pressure (HR per
SD increase $1.39,95 \%$ CI 1.32-1.47, $P<0.0001)$, but stronger than that of physical activity (HR per SD reduction $1.04,95 \%$ CI $0.991-1.09, P=0.1)$.

Among individuals with cardiovascular disease, and among those with a condition or disease that is not of a cardiovascular nature (such as cancer or respiratory disease), grip strength was inversely associated with risk of death. In their study report, the investigators note that "the increased risk of incident cardiovascular disease associated with lower grip strength is insufficient to account for its more profound increased risk for cardiovascular death". They believe their findings instead indicate that "low grip strength is associated with increased susceptibility to cardiovascular death in people who do develop cardiovascular disease". Similarly, because they found that grip strength is inversely associated with risk of death not attributable to a cardiovascular cause, but not associated with various noncardiovascular diseases, they say that their findings indicate that "low muscle strength predisposes to a fatal outcome if these noncardiovascular diseases develop".

Dr Darryl Leong, first author on the study report, concludes that "grip strength could be an easy and inexpensive test to assess an individual's risk of death and cardiovascular disease". By way of caution, the investigators point out that "the observational nature of this study does not allow us to make strong conclusions on the causal role of muscular strength in death or cardiovascular disease". Dr Leong also notes that "further research is needed to establish whether efforts to improve muscle strength are likely to reduce an individual's risk of death and cardiovascular disease".

Bryony M. Mearns 